\title{
A Reconciliation of Viscous and Inviscid Approaches to Computing Locomotion of Deforming Bodies
}

\author{
J.D. Eldredge
}

Received: 13 May 2009 / Accepted: 12 July 2009 / Published online: 25 July 2009

(C) The Author(s) 2009. This article is published with open access at Springerlink.com

\begin{abstract}
We present a formulation for coupled solutions of fluid and body dynamics in problems of biolocomotion. This formulation unifies the treatment at moderate to high Reynolds number with the corresponding inviscid problem. By a viscous splitting of the Navier-Stokes equations, inertial forces from the fluid are distinguished from the viscous forces, and the former are further decomposed into contributions from body motion in irrotational fluid and ambient fluid vorticity about an equivalent stationary body. In particular, the added mass of the fluid is combined with the intrinsic inertia of the body to allow for simulations of bodies of arbitrary mass, including massless or neutrally buoyant bodies. The resulting dynamical equations can potentially illuminate the role of vorticity in locomotion, and the fundamental differences of locomotion in real and perfect fluids.
\end{abstract}

Keywords Biolocomotion - Fluid-structure interaction - Computational fluid dynamics

\section{Introduction}

There has been a recent surge in computational investigations of biological and biologically-inspired locomotion in fluids. Such studies, whether focused on aerial or aquatic applications, have been motivated not only by scientific and technological curiosity, but also by the inherent challenge they present to numerical

J.D. Eldredge ( $\varangle)$

Mechanical \& Aerospace Engineering,

University of California, Los Angeles, CA 90095, USA

e-mail: eldredge@seas.ucla.edu methods. This paper is particularly concerned with studies of locomotion either at finite Reynolds number or in inviscid fluids. By the latter, we refer to locomotion in fluids in which the mechanism for vorticity production is either absent or restricted to a finite number of sharp edges (via the Kutta condition). Regardless of whether the fluid is viscous or inviscid, there are two unifying aspects to such problems: (1) The central goal is to simultaneously solve for the evolution of the body and the fluid, and (2) inertial forces-arising from both the body and the fluid-play an important role.

Indeed, much can be learned about the nature of locomotion by comparing the self-propulsion of the same system in both settings $[1,2]$. However, the computational approaches to studying inviscid and finite Reynolds number propulsion have evolved separately. Finite Reynolds number studies, for the most part, have relied on a grid-based Navier-Stokes solver and a straightforward coupling between flow and body dynamical solvers: interface forces from one solver (usually the fluid solver) are sent to the other, and kinematical conditions at the interface are returned in the opposite direction. Stability is ensured by implicit or semi-implicit time marching, with some iteration necessary in each step to achieve consistency between the fluid and the body. There are deficiencies in the approach, however. Firstly, the algorithm can result in severe stability restrictions if the body's own inertial forces are significantly weaker than those of the fluid. This can occur, for example, in slender bodies or membranous surfaces in water. Secondly, the corresponding inviscid problem cannot generally be simulated by the basic grid-based flow solver without vorticity production-even in the absence of its physical mechanism—at solid-fluid interfaces (e.g. [2]), 
and the resulting numerical dissipation can unnaturally influence the locomotion.

A number of recent studies have convincingly demonstrated that locomotion in inviscid flows is best studied within the framework of geometric mechanics, with the help of a boundary element solver to compute the potential flow [3-6]. This approach essentially leads to a view of locomotion as a natural consequence of global conservation laws for the fluid-body system, and produces an attractively clean algorithm for computing the locomotion, with a global mass matrix consisting of both intrinsic and added inertia [5]. A distinct advantage of this form of the equations is that it places no restrictions on the intrinsic mass of the bodies; in contrast, many previous formulations are challenged by special cases of massless $[7,8]$ or neutrally buoyant bodies $[9,10]$. Unfortunately, the geometric mechanics framework is not readily extendible to problems of finite Reynolds number. However, we will show in this paper that the dynamical equations for a body in viscous flow can be formulated such that, for zero viscosity, they reduce in a straightforward way to those obtained from geometric mechanics.

This formulation is based on the viscous splitting of the Navier-Stokes equations, argued rigorously by Chorin et al. [11]. The core idea of this concept is that each time step can be divided into substeps: one devoted to inviscid convection of existing vorticity, another devoted to viscous diffusion in a stationary fluid, and a final one in which new vorticity is produced to enforce the no-slip condition. Our extension inserts the dynamical/kinematical update of the body configuration in this sequence, and recognizes that an inherently inviscid locomotion problem exists within the 'cracks' of the full viscous problem. It should be noted that this paper condenses and refines an earlier version of the coupling algorithm presented in Eldredge [12].

\section{Methodology}

This paper will focus on two-dimensional problems, but the ideas can be readily extended to three-dimensional configurations. We take the density of the fluid to be unity and the generated flows to be incompressible. Multiple bodies may be present, and surface integrals in the following should be interpreted as summations over all bodies' surfaces.

Regardless of whether the flow is inviscid or viscous, the total vorticity of the global system of bodies and fluid is constrained by Kelvin's circulation theorem [13],

$\frac{\mathrm{d}}{\mathrm{d} t}\left(\boldsymbol{\Gamma}_{\omega}+\boldsymbol{\Gamma}_{\gamma}+\boldsymbol{\Gamma}_{b}\right)=0$,

where the total vorticities are defined, respectively, as

$\boldsymbol{\Gamma}_{\omega}=\int_{A_{f}} \omega \mathrm{d} A, \quad \boldsymbol{\Gamma}_{\gamma}=\oint_{S} \boldsymbol{\gamma} \mathrm{d} s \quad \boldsymbol{\Gamma}_{b}=\oint_{S} \boldsymbol{n} \times \boldsymbol{u}_{b} \mathrm{~d} s$.

The first integral accounts for the total vorticity in the fluid region $A_{f}$, the second represents the total strength of a vortex sheet on the body surface $S$, and the third is the total vorticity associated with the surface velocity, $\boldsymbol{u}_{b}$, where $\boldsymbol{n}$ is the surface normal directed into the fluid. It is noted that the strength of the vortex sheet on the body surface is equal to the discontinuous difference between tangential fluid and body velocities, $\boldsymbol{\gamma}=$ $\boldsymbol{n} \times\left(\boldsymbol{u}_{f}-\boldsymbol{u}_{b}\right)$, where $\boldsymbol{u}_{f}$ is the velocity on the surface when approached from the fluid side. Collectively, the last two terms in equation (1) account for the total 'bound circulation' of the bodies (the net cyclic constant associated with tight counter-clockwise loops around the bodies) in an inviscid problem.

The force exerted by the fluid on a body (or set of bodies) in viscous or inviscid flow can be written in terms of rates of change of linear impulse [13, 14],

$\boldsymbol{F}^{f}=-\frac{\mathrm{d}}{\mathrm{d} t}\left(\boldsymbol{P}_{\omega}+\boldsymbol{P}_{\gamma}+\boldsymbol{P}_{b}\right)$,

where the impulses are defined, respectively, as

$$
\begin{aligned}
\boldsymbol{P}_{\omega} & =\int_{A_{f}} \boldsymbol{x} \times \omega \mathrm{d} A, \quad \boldsymbol{P}_{\gamma}=\oint_{S} \boldsymbol{x} \times \boldsymbol{\gamma} \mathrm{d} s, \\
\boldsymbol{P}_{b} & =\oint_{S} \boldsymbol{x} \times\left(\boldsymbol{n} \times \boldsymbol{u}_{b}\right) \mathrm{d} s .
\end{aligned}
$$

The torque exerted on the body (about the origin) has a similar form,

$\boldsymbol{\tau}^{f}=-\frac{\mathrm{d}}{\mathrm{d} t}\left(\boldsymbol{\Pi}_{\omega}+\boldsymbol{\Pi}_{\gamma}+\boldsymbol{\Pi}_{b}\right)$,

where

$$
\begin{aligned}
\boldsymbol{\Pi}_{\omega} & =\frac{1}{2} \int_{A_{f}} \boldsymbol{x} \times(\boldsymbol{x} \times \boldsymbol{\omega}) \mathrm{d} A, \quad \boldsymbol{\Pi}_{\gamma}=\frac{1}{2} \oint_{S} \boldsymbol{x} \times(\boldsymbol{x} \times \boldsymbol{\gamma}) \mathrm{d} s, \\
\boldsymbol{\Pi}_{b} & =\frac{1}{2} \oint_{S} \boldsymbol{x} \times\left[\boldsymbol{x} \times\left(\boldsymbol{n} \times \boldsymbol{u}_{b}\right)\right] \mathrm{d} s .
\end{aligned}
$$

These equations (3) and (5) form the basis for our derivation. We will address the inviscid and viscous problems separately, but will arrive at a common form. 
Inviscid problems The persistent presence of the vortex sheet on the body surface is consistent with the enforcement of the no-flow-through condition, which is the only appropriate kinematic constraint in an inviscid problem; the strength of the sheet can be determined by solving an integral equation arising from this condition, subject to the additional constraint of Kelvin's circulation theorem [15]. This sheet strength can be linearly decomposed into contributions from fluid vorticity and body motion, $\boldsymbol{\gamma}=\boldsymbol{\gamma}_{\omega}+\boldsymbol{\gamma}_{b}$. In other words, $\boldsymbol{\gamma}_{\omega}$ is the strength of the surface vortex sheet in reaction to ambient fluid vorticity surrounding a stationary body, and $\boldsymbol{\gamma}_{b}$ is the sheet associated with a body in motion in an irrotational fluid. The individual integral equations for $\boldsymbol{\gamma}_{b}$ and $\boldsymbol{\gamma}_{\omega}$ are each subject to Kelvin's circulation theorem, e.g. $\oint_{S} \boldsymbol{\gamma}_{\omega} \mathrm{d} s=-\int_{A_{f}} \omega \mathrm{d} A$ and $\oint_{S} \boldsymbol{\gamma}_{b} \mathrm{~d} s=$ $-\oint_{S} \boldsymbol{n} \times \boldsymbol{u}_{b} \mathrm{~d} s$ if the global circulation is initially zero.

This decomposition leads to natural splittings of the sheet impulses, $\boldsymbol{P}_{\gamma}=\boldsymbol{P}_{\gamma \omega}+\boldsymbol{P}_{\gamma b}$ and $\boldsymbol{\Pi}_{\gamma}=\boldsymbol{\Pi}_{\gamma \omega}+$ $\boldsymbol{\Pi}_{\gamma b}$. We then note that the sum of the impulses associated strictly with body motion can be manipulated further, viz.,

$$
\begin{aligned}
\boldsymbol{P}_{\gamma b}+\boldsymbol{P}_{b} & =\oint_{S} \boldsymbol{x} \times\left(\boldsymbol{\gamma}_{b}+\boldsymbol{n} \times \boldsymbol{u}_{b}\right) \mathrm{d} s \\
& =\oint_{S} \boldsymbol{x} \times\left(\boldsymbol{n} \times \nabla \varphi_{b}\right) \mathrm{d} s .
\end{aligned}
$$

The scalar potential $\varphi_{b}$ is that which results from solving the potential flow problem in $A_{f}$ due to body motion in an irrotational fluid. Provided that there is no bound circulation associated with any constituent body-so that cyclic constants vanish-the last integral can be written in the familiar form $-\oint \boldsymbol{n} \varphi_{b} \mathrm{~d} s$. The angular impulses can be similarly manipulated into $-\oint \boldsymbol{x} \times \boldsymbol{n} \varphi_{b} \mathrm{~d} s$. This potential flow problem is linear in the components of surface velocity—rigid body motion plus some parametrization of surface shape changeso the scalar potential can be linearly decomposed accordingly $[6,16,17]$. From these forms, an added mass representation can be identified, which we write generically as

$$
\left(\begin{array}{c}
\boldsymbol{P}_{\gamma b}+\boldsymbol{P}_{b} \\
\boldsymbol{\Pi}_{\gamma b}+\boldsymbol{\Pi}_{b}
\end{array}\right)=\boldsymbol{M} \cdot \boldsymbol{U}
$$

In these equations, the vector $\boldsymbol{U}$ consists of the rigid body components of velocity (translational and rotational), as well as the rates of change of the parameters that describe the body shape. Figure 1 depicts two examples and their associated time-varying degrees of freedom. In the first, an articulated system of linked rigid bodies contains three rigid components $\left(X_{0}, Y_{0}\right.$ and $\alpha_{0}$ ) and two shape parameters (the angles $\theta_{1}$ and $\left.\theta_{2}\right)$. That is, $\boldsymbol{U}=\left(\dot{X}_{0}, \dot{Y}_{0}, \dot{\alpha}_{0}, \dot{\theta}_{1}, \dot{\theta}_{2}\right)^{T}$. In the second example, consisting of a deforming fish-like shape with a time-varying centerline curvature distribution $\kappa$, the same three rigid components are augmented by a single shape parameter $S$ controlling the phase of the curvature ( $K$ and $\lambda$ are the time-invariant amplitude and wavelength, respectively). Thus, $\boldsymbol{U}=\left(\dot{X}_{0}, \dot{Y}_{0}, \dot{\alpha}_{0}, \dot{S}\right)^{T}$. Each column of the matrix $\boldsymbol{M}$ requires the solution of a boundary integral problem and represents the instantaneous linear and angular impulse associated with a unit value of the corresponding entry in $\boldsymbol{U}$ and zeros in the other entries.

Thus, we arrive at the forms of fluid force and torque appropriate for inviscid problems,

$$
\left(\begin{array}{c}
\boldsymbol{F}^{f} \\
\boldsymbol{\tau}^{f}
\end{array}\right)=-\frac{\mathrm{d}}{\mathrm{d} t}\left(\begin{array}{c}
\boldsymbol{P}_{\omega}+\boldsymbol{P}_{\gamma \omega} \\
\boldsymbol{\Pi}_{\omega}+\boldsymbol{\Pi}_{\gamma \omega}
\end{array}\right)-\frac{\mathrm{d}}{\mathrm{d} t}(\boldsymbol{M} \cdot \boldsymbol{U})
$$

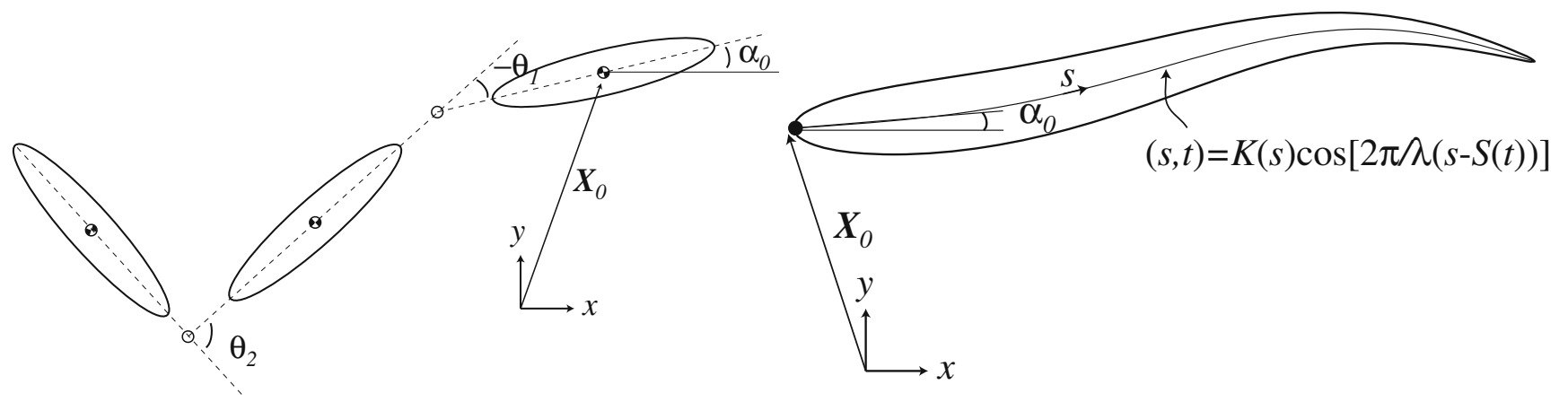

Fig. 1 Examples of parameterized shape deformation for biolocomotion studies 
The dynamical equations for the body can be written as

$\frac{\mathrm{d}}{\mathrm{d} t}\left[\left(\boldsymbol{M}_{b}+\boldsymbol{M}\right) \cdot \boldsymbol{U}\right]=-\frac{\mathrm{d}}{\mathrm{d} t}\left(\begin{array}{c}\boldsymbol{P}_{\omega}+\boldsymbol{P}_{\gamma \omega} \\ \boldsymbol{\Pi}_{\omega}+\boldsymbol{\Pi}_{\gamma \omega}\end{array}\right)+\left(\begin{array}{c}\boldsymbol{F}^{e x t} \\ \boldsymbol{\tau}^{e x t}\end{array}\right)$,

where $\boldsymbol{M}_{b}$ is the intrinsic inertia matrix of the body, and $\boldsymbol{F}^{e x t}$ and $\boldsymbol{\tau}^{e x t}$ are the external force and torque exerted on the body. The impulses $\boldsymbol{P}_{\gamma \omega}$ and $\boldsymbol{\Pi}_{\gamma \omega}$ require an additional boundary integral solution. Equations (10) are integrated in tandem with those for the body configuration and for the vorticity dynamics (if any).

Viscous problems When viscosity is present, the noflow-through kinematic condition is augmented by the no-slip condition on the body surface. Lighthill [18] postulated that this extra condition is consistent with the creation of new vorticity at the surface, by the flux of the vortex sheet associated with the no-flowthrough condition into the adjacent fluid. This has been used as the basis for a numerical algorithm with vortex particle methods (e.g. $[12,19,20])$ and a Cartesian grid method [21]. For the purposes of the present paper, we can regard each timestep as split into two subseps: in the first, fluid vorticity evolves by convection and the body evolves by its own dynamics, but their independent evolution leaves a spurious slip velocity on the body surface; this slip is then annihilated in the second substep by diffusing the vortex sheet into the fluid (while the fluid and body remain stationary). The vortex sheet is introduced into the fluid by solving a linear diffusion problem with the Neumann boundary condition $v \partial \omega / \partial n=-\gamma / \Delta t$, where $v$ is the kinematic viscosity, $\boldsymbol{\gamma}$ is the strength of the vortex sheet associated with spurious slip, and $\Delta t$ is the (vanishingly-small) time interval over which the sheet is diffused [19].

In lieu of a formal proof, the development of the governing equations is merely sketched out here; it is likely that convergence can be rigorously proved using product formulas similar to those that ground the viscous vortex particle method in functional analysis [11]. Let us write equation (3) as a limit of $\Delta t \rightarrow 0$, but explicitly introduce the intermediate stage ()$^{*}$ in this time interval at which the spurious vortex sheet exists on the surface. Before doing so, we note that this spurious sheet (and the associated slip velocity) is eliminated at the end of any time step, so $\boldsymbol{P}_{\gamma}^{n}=0$ at any time level $t^{n}$. Thus,

$$
\begin{aligned}
\boldsymbol{F}^{f}= & -\lim _{\Delta t \rightarrow 0} \frac{1}{\Delta t}\left[\left(\boldsymbol{P}_{\omega}^{n+1}-\boldsymbol{P}_{\omega}^{*}+\boldsymbol{P}_{\omega}^{*}-\boldsymbol{P}_{\omega}^{n}\right)\right. \\
& \left.+\left(\boldsymbol{P}_{b}^{n+1}-\boldsymbol{P}_{b}^{*}+\boldsymbol{P}_{b}^{*}-\boldsymbol{P}_{b}^{n}\right)\right] .
\end{aligned}
$$

The intermediate body impulse $\boldsymbol{P}_{b}^{*}$ is identical to its final value $\boldsymbol{P}_{b}^{n+1}$, since the body remains stationary during the second substep. On the other hand, the splitting of the fluid vorticity impulse allows us to distinguish an essentially inviscid contribution to its change in the first substep from a viscous contribution in the second substep. In fact, the first of these contributions is identical to the corresponding term in the inviscid problem; we label this as $-\mathrm{d} \boldsymbol{P}_{\omega} /\left.\mathrm{d} t\right|_{i}$. The second (viscous) contribution can be manipulated into a surface integral form by bringing the time derivative into the integral, and making use of the diffusion equation and the divergence theorem,

$-\left.\frac{\mathrm{d} \boldsymbol{P}_{\omega}}{\mathrm{d} t}\right|_{v}=\oint_{S} \boldsymbol{x} \times v \frac{\partial \boldsymbol{\omega}}{\partial n} \mathrm{~d} s-v \oint_{S} \boldsymbol{n} \times \boldsymbol{\omega} \mathrm{d} s$.

The last of these two integrals is the force due to viscous shear stress, which we label as $\boldsymbol{F}_{v}^{f}$. The first integral can be re-written in terms of the impulse of the spurious vortex sheet by virtue of the Neumann boundary condition on vorticity, which leads to

$-\left.\frac{\mathrm{d} \boldsymbol{P}_{\omega}}{\mathrm{d} t}\right|_{v}=-\frac{1}{\Delta t} \boldsymbol{P}_{\gamma}^{*}+\boldsymbol{F}_{v}^{f}$

However, as in the inviscid case, the strength of this vortex sheet can be decomposed into parts contributed by fluid vorticity and body motion, $\boldsymbol{\gamma}=\boldsymbol{\gamma}_{\omega}+\boldsymbol{\gamma}_{b}$ (again, with each subject to Kelvin's circulation theorem). Furthermore, since the spurious slip is absent at the beginning and end of the full time interval, then $\boldsymbol{P}_{\gamma \omega}^{n}=$ $-\boldsymbol{P}_{\gamma b}^{n}$. Thus, the term with the impulse of the intermediate vortex sheet can be written, in the limit of vanishing time interval, as

$$
\begin{aligned}
-\lim _{\Delta t \rightarrow 0} \frac{1}{\Delta t} \boldsymbol{P}_{\gamma}^{*}= & -\lim _{\Delta t \rightarrow 0} \frac{1}{\Delta t}\left(\boldsymbol{P}_{\gamma \omega}^{*}-\boldsymbol{P}_{\gamma \omega}^{n}\right) \\
& -\frac{1}{\Delta t}\left(\boldsymbol{P}_{\gamma b}^{*}-\boldsymbol{P}_{\gamma b}^{n}\right) \\
= & -\frac{\mathrm{d}}{\mathrm{d} t}\left(\boldsymbol{P}_{\gamma \omega}+\boldsymbol{P}_{\gamma b}\right)
\end{aligned}
$$

A similar process can be carried out for the fluid torque. Thus, bringing all terms together, we arrive at a form for the fluid force and torque in a viscous problem that is structurally analogous to the inviscid problem,

$$
\left(\begin{array}{c}
\boldsymbol{F}^{f} \\
\boldsymbol{\tau}^{f}
\end{array}\right)=-\left.\frac{\mathrm{d}}{\mathrm{d} t}\right|_{i}\left(\begin{array}{c}
\boldsymbol{P}_{\omega}+\boldsymbol{P}_{\gamma \omega} \\
\boldsymbol{\Pi}_{\omega}+\boldsymbol{\Pi}_{\gamma \omega}
\end{array}\right)-\frac{\mathrm{d}}{\mathrm{d} t}(\boldsymbol{M} \cdot \boldsymbol{U})+\left(\begin{array}{c}
\boldsymbol{F}_{v}^{f} \\
\boldsymbol{\tau}_{v}^{f}
\end{array}\right),
$$


where the viscous shear stress contributions are

$$
\begin{aligned}
\boldsymbol{F}_{v}^{f} & =-v \oint_{S} \boldsymbol{n} \times \boldsymbol{\omega} \mathrm{d} s, \\
\boldsymbol{\tau}_{v}^{f} & =-v \oint_{S}\left[\boldsymbol{x} \times(\boldsymbol{n} \times \boldsymbol{\omega})+2 \boldsymbol{n} \times \boldsymbol{u}_{b}\right] \mathrm{d} s .
\end{aligned}
$$

The added mass contributions have now been explicitly drawn out of the overall force. As in the inviscid case, these can be grouped together with the intrinsic body inertia to form the left-hand side of the dynamical equations for the body, leading to our final result,

$$
\begin{aligned}
\frac{\mathrm{d}}{\mathrm{d} t}\left[\left(\boldsymbol{M}_{b}+\boldsymbol{M}\right) \cdot \boldsymbol{U}\right] \\
\quad=-\left.\frac{\mathrm{d}}{\mathrm{d} t}\right|_{i}\left(\begin{array}{l}
\boldsymbol{P}_{\omega}+\boldsymbol{P}_{\gamma \omega} \\
\boldsymbol{\Pi}_{\omega}+\boldsymbol{\Pi}_{\gamma \omega}
\end{array}\right)+\left(\begin{array}{c}
\boldsymbol{F}^{e x t}+\boldsymbol{F}_{v}^{f} \\
\boldsymbol{\tau}^{e x t}+\boldsymbol{\tau}_{v}^{f}
\end{array}\right) .
\end{aligned}
$$

This formulation shows clearly that the finite Reynolds number locomotion problem differs from the inviscid problem both directly-through the shear stress contributions-and indirectly-through the continuous creation of vorticity.

\section{Conclusions}

This paper has presented dynamical equations for locomotion that unifies the inviscid and viscous approaches to the problem. These equations allow simulations of bodies with arbitrary mass-including massless and neutrally buoyant bodies-which has been impossible in previous formulations, and can potentially shed light on the role of vorticity in locomotion. It should be noted that, though the development of the viscous dynamical equations has been motivated by the vortex particle method, the equations can also be used with other numerical fluid schemes, provided that additional consideration is given for computing the added inertia coefficients and the inviscid rates of change of impulse. The latter can be re-written in forms more conducive to a specific solver. It is also possible to develop analogous dynamical equations for systems with elasticity; this will be the subject of a forthcoming paper.

Open Access This article is distributed under the terms of the Creative Commons Attribution Noncommercial License which permits any noncommercial use, distribution, and reproduction in any medium, provided the original author(s) and source are credited.

\section{References}

1. Eldredge JD (2006) Numerical simulations of undulatory swimming at moderate reynolds number. Bioinspir Biomim 1(4):S19-S24

2. Borazjani I, Sotiropoulos F (2008) Numerical investigation of the hydrodynamics of carangiform swimming in the transitional and inertial flow regimes. J Exp Biol 211:1541-1558

3. Kelly SD (1998) The mechanics and control of robotic locomotion with applications to aquatic vehicles. $\mathrm{PhD}$ thesis, California Institute of Technology

4. Shashikanth BN, Marsden JE, Burdick JW, Kelly SD (2002) The Hamiltonian structure of a two-dimensional rigid circular cylinder interacting dynamically with $\mathrm{N}$ point vortices. Phys Fluids 14(3):1214-1227

5. Kanso E, Marsden JE, Rowley CW, Melli-Huber JB (2005) Locomotion of articulated bodies in a perfect fluid. J Nonlinear Sci 15(4):255-289

6. Kanso E, Oskouei BG (2008) Stability of a coupled bodyvortex system. J Fluid Mech 600:77-94

7. Carling J, Williams TL, Bowtell G (1998) Self-propelled anguilliform swimming: simultaneous solutions of the twodimensional Navier-Stokes equations and Newton's laws of motion. J Exp Biol 201:3143-3166

8. Kern S, Koumoutsakos P (2006) Simulations of optimized anguilliform swimming. J Exp Biol 209:4841-4857

9. Andersen A, Pesavento U, Wang ZJ (2005) Unsteady aerodynamics of fluttering and tumbling plates. J Fluid Mech 541:65-90

10. Spagnolie SE, Shelley MJ (2009) Shape-changing bodies in fluid: hovering, ratcheting, and bursting. Phys Fluids 21(1):013103

11. Chorin AJ, Hughes TJR, McCracken MF, Marsden JE (1978) Product formulas and numerical algorithms. Commun Pure Appl Math 31(2):205-256

12. Eldredge JD (2008) Dynamically coupled fluid-body interactions in vorticity-based numerical simulations. J Comput Phys 227(21):9170-9194

13. Wu JC (1981) Theory for aerodynamic force and moment in viscous flows. AIAA J 19(4):432-441

14. Saffman PG (1992) Vortex dynamics. Cambridge University Press, Cambridge

15. Zhang LJ, Eldredge JD (2009) A viscous vortex particle method for deforming bodies with application to biolocomotion. Int J Numer Methods Fluids 59:1299-1320

16. Lamb H (1932) Hydrodynamics, 6th edn. Cambridge University Press, Cambridge

17. Xiong H, Kelly SD (2008) Self-propulsion of a deformable joukowski foil in a perfect fluid with vortex shedding. J Nonlinear Sci (under review)

18. Lighthill MJ (1961) Introduction: boundary layer theory. In: Rosenhead L (ed) Laminar boundary layers. Clarendon Press, Clarendon

19. Koumoutsakos P, Leonard A, Pepin F (1994) Boundary conditions for viscous vortex methods. J Comput Phys 113(1): 52-61

20. Eldredge JD (2007) Numerical simulation of the fluid dynamics of $2 \mathrm{D}$ rigid body motion with the vortex particle method. J Comput Phys 221(2):626-648

21. Russell D, Wang ZJ (2003) A cartesian grid method for modeling multiple moving objects in 2D incompressible viscous flow. J Comput Phys 191:177-205 